The Astrophysical Journal, 187 :L67-L71, 1974 January 15

(c) 1974. The American Astronomical Society. All rights reserved. Printed in U.S.A.

\title{
RADIATIVE TRANSFER, EXCITATION, AND COOLING OF MOLECULAR EMISSION LINES (CO and CS)
}

\author{
N. Z. SCOVILle* AND P. M. Solomon† \\ School of Physics and Astronomy, University of Minnesota \\ Received 1973 October 17; revised 1973 November 15
}

\begin{abstract}
We consider the radiative transfer of molecular lines in interstellar clouds having flow velocities large compared with random motions. The equilibrium level populations of $\mathrm{CO}$ and $\mathrm{CS}$ are calculated including the effects of both self-radiation (radiative trapping) and collisions with hydrogen molecules (using recently measured cross-sections). Analytic expressions are also developed for the excitation of a two-level molecule. Because of the velocity gradients in the cloud, the observed emission will originate not only from the near boundary but also from interior regions where excitation is greatly enhanced by scattered photons. This ability to see into the clouds qualitatively accounts for the general similarities of the different line profiles in individual clouds and considerably reduces the density of collisional particles needed to account for the observed excitation. Remarkably, even if $A>C$ but $\tau>1$, the excitation temperature (and the observed intensity) depends on the molecular density but is totally independent of the spontaneous rate $A$. The rate of gas cooling by $\mathrm{CO}$ molecules in clouds of moderate density $\left(n_{\mathrm{H}_{2}} \sim 10^{3}\right.$ $\left.\mathrm{cm}^{-3}\right)$ is high $\left(\sim 10^{-22}\right.$ ergs $\left.\mathrm{cm}^{-3} \mathrm{~s}^{-1}\right)$ even when the important cooling transitions $(J=3 \rightarrow 2$ and higher) are optically thick. These results are applicable to either cloud collapse or expansion.

Subject headings: molecules, interstellar - radiative transfer
\end{abstract}

\section{INTRODUCTION}

To date more than 100 millimeter-wavelength rotational transitions of molecules have been observed in emission from numerous molecular clouds throughout the Galaxy. The intensities of the lines run from the present limit of detectability $\left(\sim 0.1^{\circ} \mathrm{K}\right)$ up to about $70^{\circ} \mathrm{K}$ for the strongest CO $J=1 \rightarrow 0$ line, with the line widths $\left(1-40 \mathrm{~km} \mathrm{~s}^{-1}\right)$ always much larger than thermal $\left(0.1 \mathrm{~km} \mathrm{~s}^{-1}\right)$. The relatively high intensities of several molecular isotope lines (e.g., ${ }^{13} \mathrm{CO}$ and $\mathrm{C}^{34} \mathrm{~S}$ ) indicate high optical depths in many of the transitions. Here we discuss the non-LTE formation of these lines and emphasize that the processes important in forming optically thick transitions are strongly dependent on the assumed mechanism of line broadening-either local turbulence or large-scale gas flows. In the first case the observed excitation is determined primarily by a balance between collisions (with $\mathrm{H}_{2}$ ) and radiative processes at the near edge of the cloud (at $\tau \sim 1$ ). The presence of large velocity gradients will alter this picture drastically; for then the observed radiation is contributed equally by molecules along the entire line of sight, in the cloud interior, where the excitation may be greatly enhanced by absorption of trapped line photons.

The assumption of local line broadening, implicit to all existing analyses of data, is implausible considering the rapid damping of small-scale, hypersonic turbulence. And, from the observational viewpoint, the great similarity of line profiles for molecules with very different optical depths would imply unbelievable constancy of the turbulence at different distances into the cloud. In the same regions obvious profile changes are noted across the cloud face, and the condition of balance between collisions and spontaneous decays would imply densities ranging from $10^{3}$ to $10^{8} \mathrm{~cm}^{-3}$ (see Scoville and Solomon 1973).

In the following sections we treat the line transfer, molecular excitation, and cooling in a region having line-ofsight flow velocities larger than local random velocities. In this approximation, the equilibrium level populations of $\mathrm{CO}$ and CS including both collisions and self-radiation are easily calculated since the radiative transfer is a local problem. Indeed, although full 10-level numerical calculations were carried out for the results shown in the figures, we have also obtained simple analytic expressions for the two-level molecule which illustrate well the basic processes of excitation. A more extensive expose of this theory and detailed comparisons with observations are the subjects of a second paper (Scoville and Solomon 1974).

\section{RADIATIVE TRANSFER}

Our model for line formation is adopted from the solution obtained by Lucy (1971) for resonance lines in expanding atmospheres. The line-of-sight flow velocities (characterized by a velocity gradient $d v / d z$ ) are assumed to be much greater than the local random velocities $v_{t}$ (thermal or microturbulent). Since each point in the cloud is indistinguish-

* Present address: Astronomy Department, California Institute of Technology.

†.Present address: Institute for Advanced Study, Princeton, New Jersey. 
able from any other (except the outermost layer of $\Delta z<v_{t} / d v / d z$ ) as long as $d v / d z$ does not change sign, the radiative transfer becomes a local problem and analytic solutions exist for the intensity and energy density. We adopt a plane-parallel geometry for the cloud, microwave background radiation with intensity $I_{o}$ at the boundaries, and assume local complete frequency redistribution of scattered photons. The energy density (averaged over the local absorption profile), source function, and optical depth are given by

and

$$
\begin{gathered}
U_{\nu^{\prime}}=\frac{4 \pi S}{c}\left[1-\frac{1}{3 \tau}\left(1-e^{-3 \tau}\right)\right]+\frac{4 \pi}{c} \frac{I_{0}}{3 \tau}\left[1-e^{-3 \tau}\right], \\
S=\frac{2 h \nu^{\prime 3}}{c^{2}} \frac{1}{g_{u l} n_{l} / n_{u}-1},
\end{gathered}
$$

The number density $n\left[\mathrm{~cm}^{-3}\right]$, excitation temperature $T_{l u}$, and statistical-weight ratio $g_{u l}$ of the molecules in the upper and lower levels are subscripted by $u$ and $l ; T_{o} \equiv h \nu / k$ is the temperature equivalent of the transition energy, and $\alpha \equiv g_{u l} c^{3} /\left(8 \pi \nu^{3} d v / d z\right)$. The term $\frac{1}{3} \tau^{-1}\left(1-e^{-3 \tau}\right)$ appearing in equation (1) represents the fraction of emitted photons which escape from the cloud; the numerical factor will of course depend on the geometry for other models. The intensity at $\nu_{0}(1-v / c)$ emitted normal to the slab, above the background radiation, is

$$
I=\left(S-I_{0}\right)\left(1-e^{-\tau}\right) \text {. }
$$

\section{MOLECULAR EXCITATION}

For both $\mathrm{CO}$ and $\mathrm{CS}$ we have solved the nine complete rate equations governing levels $J \leq 8$ plus the substantive relation in order to find the populations of the ten lowest rotational states. The cross-sections of $\mathrm{H}_{2}-\mathrm{CO}$ excitation were taken from recent laboratory measurements (up to $\Delta J=5$, Compaan et al. 1973). Use of the same cross-sections for CS will introduce small errors which are minimized by restricting our calculations to $n_{\mathrm{H}_{2}} \leq 10^{6} \mathrm{~cm}^{-3}$ where radiative transitions dominate. The rate equations, nonlinear because of the stimulated radiative terms, were linearized numerically and solved iteratively. The molecule density $n_{m}\left[\mathrm{~cm}^{-3}\right]$ and velocity gradient $d v / d z[\mathrm{~km}$ $\mathrm{s}^{-1} \mathrm{pc}^{-1}$ ] enter in the optical depth as the ratio $n_{m} / d v / d z$. This ratio together with $n_{\mathrm{H}_{2}}$ and the kinetic temperature $T_{k}$ then serve as independent variables in the calculations. The Rayleigh-Jeans antenna temperatures equivalent to the calculated intensities (eq. [4]) are plotted as contours in the $\left(\log n_{\mathrm{H}_{2}}, \log n_{m} / d v / d z\right)$-plane (figs. 1 and 2$)$. The rate of gas cooling, $\Lambda$, in line radiation was also obtained. For CO, the most important coolant, it is approximated by the power law $6 \times 10^{-29} N_{\mathrm{CO}} N_{\mathrm{H}_{2}} T_{k}^{2}$ ergs $\mathrm{cm}^{-3} \mathrm{~s}^{-1}$ for $N_{\mathrm{CO}} N_{\mathrm{H}_{2}} \leq 10^{2}$ and becomes independent of density as the cooling transitions thermalize, giving $\Lambda \sim 2 \times 10^{-27} T_{k}^{3}$ for $N_{\mathrm{CO}} N_{\mathrm{H}_{2}} \gg 10^{2}$.

Although the numerical calculations of multilevel equilibrium are needed for quantitative comparison with observational data, the qualitative features of radiative trapping are better discussed in terms of a two-level approximation. The two-level solutions also provide a basis for scaling the multilevel CO and CS calculations to other molecules.

Letting $C_{u l}$ denote the downward collision rate and neglecting the microwave background radiation (eq. [1]), we find the two-level state temperature $T_{l u}$ given by

$$
\frac{T_{l u}}{T_{0}}=T_{k} / T_{0} \llbracket\left[1+\frac{T_{k}}{T_{0}} \ln \left\{1+\frac{A_{u l}}{C_{u l}}\left[\frac{1}{3 \tau}\left(1-e^{-3 \tau}\right)\right]\right\}\right]^{-1} .
$$

If the gas is optically thin $(\tau<1)$, radiative trapping has little effect on the excitation; equation (7) then yields the well-known result in which $T_{l u}$ depends on the ratio $A_{u l} / C_{u l}$. Unless $C_{u l}>A_{u l}$, this regime bears little relevance to observations because the line intensity is proportional to $\tau T_{l u}$ and both $\tau$ and $T_{l u}$ are small.

But as the optical depth increases, scattered line photons greatly enhance a given low level of excitation provided by $\mathrm{H}_{2}$ collisions (see fig. 3). If $\tau>1$, then the intensity is $\sim T_{l u}$ and equation (5) reduces to

$$
\frac{T_{l u}}{T_{0}}=T_{k} / T_{0}\left[1+\frac{T_{k}}{T_{0}} \ln \left(1+\frac{A_{u l}}{3 \tau C_{u l}}\right)\right]^{-1} \text {. }
$$

Herein lies the important result when equation (3) is substituted for $\tau: T_{l u}$ (and thus the observed intensity) depends only on the product $\alpha \mathrm{n}_{m} \mathrm{C}_{u l}$ and is independent of $A_{u l}$. This independence of $A_{u l}$ is easily understood since both the excitation and de-excitation rates vary linearly with $A_{u l}$. Each collisional excitation is reproduced $3 \tau$ times by photon scattering before the photon is lost; the effective net radiative decay rate is therefore reduced from $A_{u l}$ to $A_{u l} / 3 \tau$. It is the total number of photons generated by collisions $\left[\mathrm{s}^{-1} \mathrm{~Hz}^{-1}\right.$ ], depending on $\alpha n_{m} C_{u l}$ and $T_{0} / T_{k}$ which determines the intensity of the emitted line. Although the explicit dependence, $T_{l u} \propto\left(\alpha n_{m} C_{u l}\right)^{1 / 2} T_{k}$, can be derived for the limit 


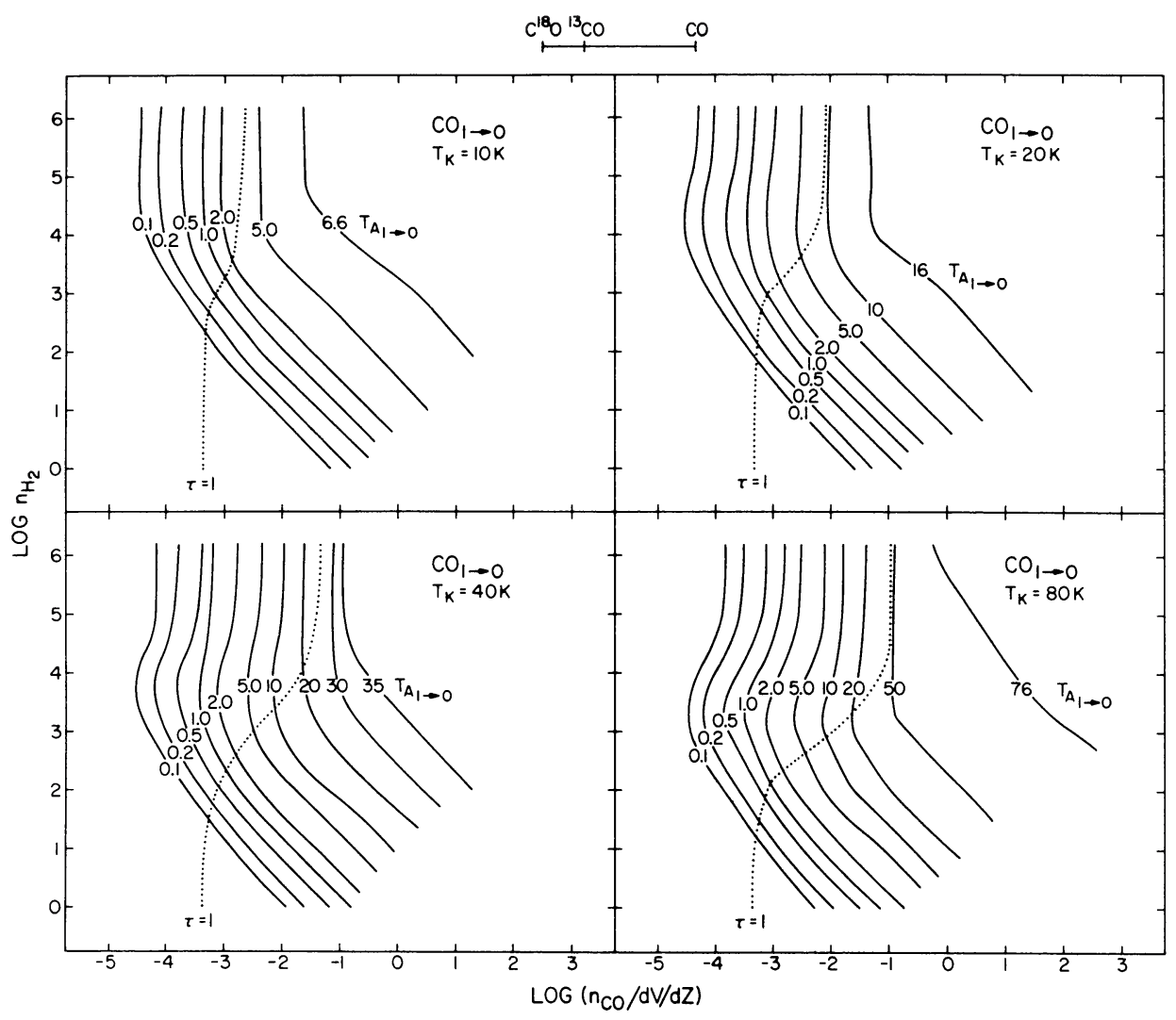

FIg. 1a.-Contours of antenna temperature $\left({ }^{\circ} \mathrm{K}\right.$ observed by a perfect antenna) in the $J=1 \rightarrow 0 \mathrm{CO}$ transition from 10-level calculations as a function of $n_{\mathrm{H}_{2}}$ and $n_{\mathrm{CO}} / d v / d z$ at four kinetic temperatures. Dotted curves indicate $\tau_{1-0}=1$ and the relative terrestrial abundances of $\mathrm{CO}$ isotopes are shown above figure. Units: $n_{\mathrm{H}_{2}}, n_{\mathrm{CO}}\left[\mathrm{cm}^{-3}\right] ; d v / d z\left[\mathrm{~km} \mathrm{~s}^{-1} \mathrm{pc}^{-1}\right]$.

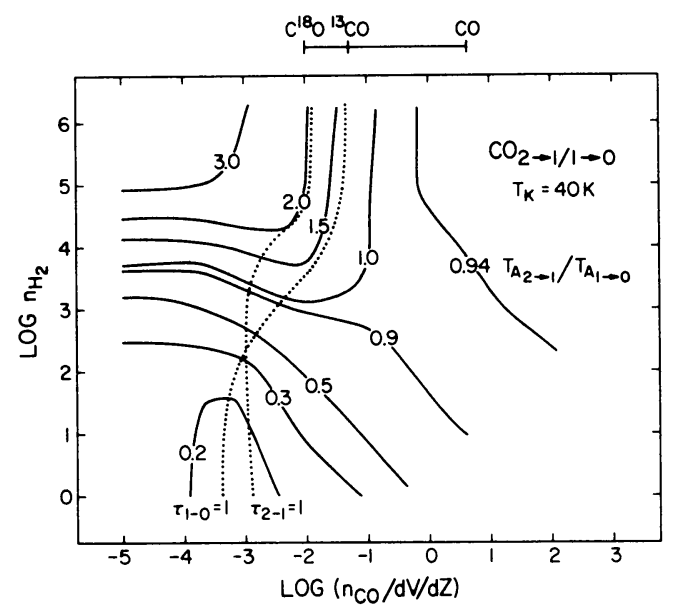

FIG. 1b.-The ratio of antenna temperatures in the $\mathrm{CO} J=2 \rightarrow 1$ and $J=1 \rightarrow 0$ transitions obtained from 10-level calculations at $T_{k}=40^{\circ} \mathrm{K}$. 


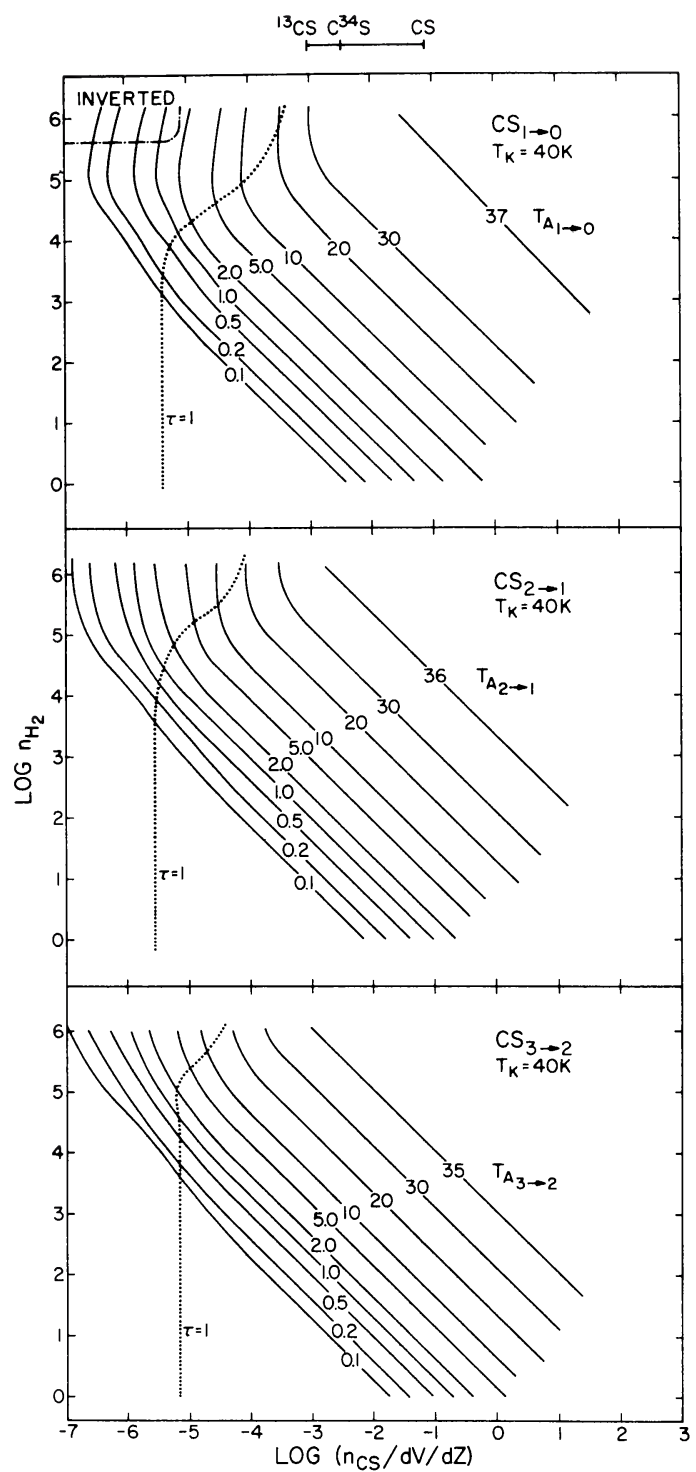

Fig. 2.-Contours of antenna temperature in the $J=1 \rightarrow 0,2 \rightarrow 1$, and $3 \rightarrow 2 \mathrm{CS}$ transitions from 10-level calculations at $T_{k}=40^{\circ} \mathrm{K}$.

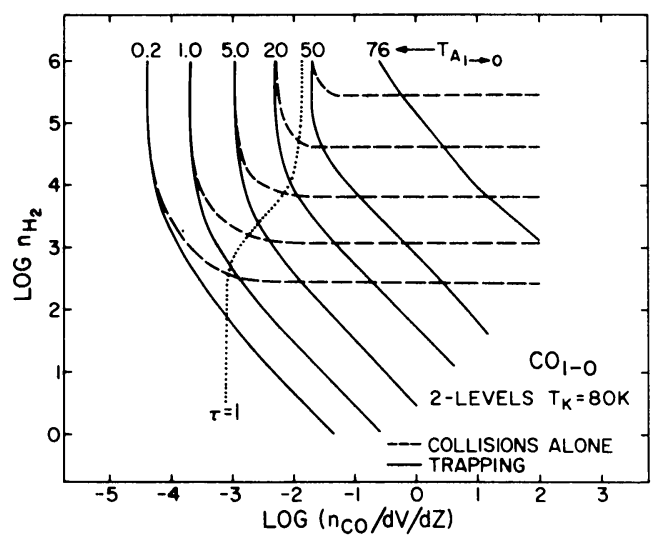

FIG. 3.-The dramatic effects of radiative trapping are demonstrated for the $J=1 \rightarrow 0$ CO transition in the two-level approximation. Dashed contours are obtained for excitation only by $\mathrm{H}_{2}$ collisions; solid contours include excitation by trapped radiation. 
$\tau C_{u l} \gg A_{u l}$ and $T_{k} \gg T_{0}, T_{l u}$, these conditions are rarely satisfied. Inspection of figures 1 and 2 suggests that $T_{l u} \propto\left(\alpha n_{m} C_{u l}\right)^{0.4 \rightarrow 0.25} T_{k}$ for more realistic parameters.

\section{CONCLUSIONS}

We briefly summarize the numerous consequences for molecular cloud physics and analysis of observations.

1. Hydrogen densities. - The collision rate and therefore density $n_{\mathrm{H}_{2}}$, necessary to account for excitation of CS, $\mathrm{HCN}, \mathrm{CH}_{3} \mathrm{OH}$, and other molecules with short lifetimes, is greatly reduced from previous estimates. In order to produce a moderate intensity line with $T_{A} \sim T_{0}$ the criterion is $\alpha n_{m} C_{u l} \sim 1$. We estimate from observations that typically $d v / d z \sim 1 \mathrm{~km} \mathrm{~s}^{-1} \mathrm{pc}^{-1}$ and therefore $n_{\mathrm{H}_{2}} \sim 3 \times 10^{4}-10^{5}$ for the dense cloud cores, with larger regions at $n_{\mathrm{H}_{2}} \sim 3 \times 10^{2}-10^{4} \mathrm{~cm}^{-3}$.

2. Line profiles.-Most observed lines, although subthermal, are optically thick. There is a wide range of observability, several orders of magnitude in $\alpha n_{m} C_{u l}$ even if the intensity is subthermal, because all intensities fall off much slower than linearly with abundance and collision rate. The relatively high intensities of $\mathrm{CO}$ lines find a natural explanation in the large $\mathrm{CO}$ abundance, about 100 times that of any other molecule. Moreover, the relatively sluggish variations of the $\mathrm{CO}$ intensity (in both spatial and frequency coordinates) indicate saturation of the $\mathrm{CO}$ excitation at a temperature close to the gas kinetic temperature (Solomon 1971).

3. Isotope ratios.-Although accurate estimates of isotope ratios will require better cloud models, the intensity ratio will always be an upper limit to the isotope ratio since the dependence on $n_{m}$ is less than linear. For example, if a molecule falls in the regime corresponding to a $\frac{1}{3}$ power law, then the true isotope ratio will be the cube of the intensity ratio.

4. Hyperfine structure.-It is possible to have hyperfine intensities close to the line strength ratios even though the line is optically thick. This is particularly important for HCN. Detailed calculations for specific models, with multilevels, indicate unsaturated hyperfine ratios for the entire subthermal regime even with $\tau \simeq 100$.

\section{REFERENCES}

Compaan, A., Langer, W. D., Eden, D., and Swinney, H. L. 1973, preprint.

Lucy, L. B. 1971, Ap.J., 163, 95.

Scoville, N. Z., and Solomon, P. M. 1973, Ap.J., 180, 31.

1974 (in preparation).

Solomon, P. M. 1971, in Charlottesville Symposium on Interstellar Molecules, "Molecules in the Galactic Environment," ed. M. Gordon and L. Snyder (New York: Wiley-Interscience).

Note added in proof. - The densities $n_{\mathrm{H}_{2}}$, given in the figures, will scale approximately inversely with the total crosssection to all levels, for collisional excitation. We have employed a total cross-section of $\sim 8 \times 10^{-16} \mathrm{~cm}^{2}(\mathrm{see}$ Compaan et al. 1973). 